\title{
Composição florística da mata ciliar no baixo rio Gramame, Paraíba, Brasil
}

\author{
Hermes de Oliveira Machado Filho ${ }^{1,2^{*}}$ \\ Thiago da Silva Farias ${ }^{1}$ \\ Alex da Silva Santos ${ }^{1}$ \\ Mateus Vieira dos Santos ${ }^{1}$ \\ Cleomar Porto Bezerra ${ }^{1,3}$ \\ José Iranildo Miranda de Melo ${ }^{4}$ \\ ${ }^{1}$ Instituto Federal de Educação, Ciência e Tecnologia da Paraíba \\ Avenida 1ํ de Maio, 720, Jaguaribe, CEP 58.015-430, João Pessoa - PB, Brasil \\ 2 Programa de Pós-Graduação em Botânica, Universidade Federal Rural de Pernambuco \\ Rua Dom Manoel de Medeiros, s/n, Dois Irmãos, CEP 52.171-900, Recife - PE, Brasil \\ 3 Programa de Pós-Graduação em Educação, Universidade Federal do Rio Grande do Norte \\ Caixa Postal 1524, Lagoa Nova, CEP 59.072-970, Natal - RN, Brasil \\ ${ }^{4}$ Universidade Estadual da Paraíba, Avenida das Baraúnas, s/n, Bairro Universitário, \\ CEP 58429-500, Campina Grande - PB, Brasil \\ * Autor para correspondência \\ hermes@ifpb.edu.br
}

Submetido em 02/09/2014

Aceito para publicação em 22/04/2015

\section{Resumo}

A mata ciliar tem uma importância ecológica e econômica fundamental para as cadeias produtivas associadas a ela. Este estudo teve por objetivo realizar um levantamento florístico de trechos da mata ciliar do rio Gramame, estado da Paraíba, Nordeste do Brasil, e analisar a similaridade florística com fragmentos de vegetação ciliar brasileiros. Foram encontradas 136 espécies pertencentes a 106 gêneros e 43 famílias. As famílias mais representativas foram: Fabaceae (19 spp.), Cyperaceae (16 spp.) e Rubiaceae (11 spp.). O hábito predominante foi o herbáceo e o espectro biológico mais bem representado foi o caméfito. Em relação à distribuição geográfica, predominaram as espécies de ampla distribuição associadas à província neotropical. Os padrões de distribuição evidenciaram baixa similaridade entre as áreas, destacando-se as espécies de ampla distribuição. A análise de similaridade apontou que a área esteve floristicamente relacionada a outras duas áreas costeiras das regiões Nordeste e Sudeste do Brasil. Apenas espécies tipicamente relacionadas aos ambientes estuarinos explicariam as conexões florísticas detectadas.

Palavras-chave: Flora; Nordeste setentrional; Veredas

\section{Abstract}

Floristic composition of the riparian forest in the lower Gramame river, Paraíba, Brazil. Riparian forest has a key ecological and economic significance to productive chains associated with it. This study aimed to conduct a floristic survey of riparian forest stretches in the Gramame river, state of Paraíba, Brazilian Northeast 
region, and analyze the floristic similarity with Brazilian riparian vegetation fragments. We found 136 species belonging to 106 genera and 43 families. The most representative families were: Fabaceae (19 spp.), Cyperaceae (16 spp.), and Rubiaceae (11 spp.). The predominant habit was herbaceous and the best represented biological spectrum was camephyte. Regarding the geographic distribution, there was a predominance of widely distributed species associated with the Neotropical province. The distribution patterns have shown a low similarity between areas, and largely distributed species stand out. Similarity analysis pointed out that the area was floristically related to other two coastal areas in the Brazilian Northeast and Southeast regions. Only species typically related to estuarine environments might explain the floristic connections detected.

Key words: Flora; Hinterlands; Northern Northeast

\section{Introdução}

A vegetação ciliar pode ser definida como aquela típica de margens ou áreas próximas a corpos aquáticos; sejam rios, lagos, represas, córregos ou várzeas, apresentando composição de espécies resistentes ou tolerantes ao encharcamento ou excesso de água no solo, sazonal ou permanentemente (KAGEYAMA et al., 2001; SOUZA et al., 2011). Essa vegetação recebe diversas denominações como mata ciliar, floresta ou mata de galeria, veredas, mata de várzea, floresta beiradeira, entre outras (KAGEYAMA et al., 2001; SOUZA et al., 2011).

As matas ciliares apresentam diversas funções e importâncias, tanto na preservação do ponto de vista ecológico quanto no aspecto econômico. Elas funcionam como reguladoras de processos erosivos (anti-assoreamento), estabilizam margens, promovem ciclagem de nutrientes, facilitam a interação solo-águafauna e promovem a estabilidade térmica dos corpos d'água (PEDRALLI; TEIXEIRA, 1997; LEANDRO; VIVEIROS, 2003; LOURES et al., 2007; ZEPEDAGÓMEZ et al., 2012).

Além de serem uma reserva natural para várias espécies, as matas ciliares garantem uma alta biodiversidade em seu meio, servindo também de corredores ecológicos, proporcionando conexões entre remanescentes de vegetação nativa (VOGEL et al., 2009) e tudo isso contribui para a diversidade como reservatório de espécies que podem ser até utilizadas para a recolonização de lugares antropizados (MARQUES et al., 2003).

Apesar de sua importância, as florestas ripárias aproximam-se da descaracterização completa em várias partes do Brasil (GIBBS et al., 1980; NICÁCIO, 2001;
LOURES et al., 2007). A urbanização, a instalação de indústrias, o aumento das fronteiras agropecuárias e a falta de uma fiscalização efetiva são os fatores que propiciam a degradação acentuada das matas ciliares (ESTEVES, 2011). Apesar da existência de legislação preservacionista desde o final do século XX (BRASIL, 1989), a Lei no 4.771, de 15 de setembro de 1965 , alterada pela Lei no 7.803 , de 18 de julho de 1989, a vegetação ciliar é submetida a ações de degradação intensas, como a retirada de madeira, a implantação de pequenos e grandes empreendimentos, agricultura desordenada, entre outros (SOUZA et al., 2008).

Nos últimos anos, estudos de levantamentos florísticos em matas ciliares foram realizados em diversos ecossistemas brasileiros como, por exemplo, na Área de Proteção Ambiental (APA) de Jericoacoara, região litorânea do Ceará (MATIAS; NUNES, 2001); em uma zona de brejo de altitude no município de Areia, Paraíba (ANDRADE et al., 2006); na floresta de igapó na Amazônia Central (SCUDELLER; SOUZA, 2009); em uma floresta ribeirinha no cerrado de Mato Grosso do Sul (BAPTISTA-MARIA et al., 2009); em planícies de inundação nos pampas gaúchos (MARCHI; JARENKOW, 2008; KILCA et al., 2011;); no semiárido do nordeste do Brasil (SILVA et al., 2012) e no cerrado de Minas Gerais (PIVARI et al., 2013), por exemplo.

As informações resultantes desses levantamentos florísticos também têm sido importantes para compreensão dos padrões biogeográficos da vegetação (BAPTISTA-MARIA et al., 2009), bem como atender as demandas de acordos internacionais como a Convenção sobre Diversidade Biológica (CBD, 2013), o Protocolo de Kyoto (UNITED NATIONS, 1998) do United Nations Framework Convention on Climate Change 
(UNFCCC) da Food and Agriculture Organization of the United Nations (FAO) e levantamentos globais como o Global Forest Resources Assessment (FAO, 2010), que obrigam os Estados signatários a disponibilizarem informações sobre o estado dessas áreas prioritárias para a conservação e restauração desses ecossistemas florestais.

Nesse contexto, o presente estudo objetivou caracterizar a composição florística de trechos da mata ciliar na bacia do baixo Rio Gramame (Paraíba, Brasil), como também detectar os tipos de hábitos, o espectro biológico, a distribuição geográfica das espécies registradas na área estudada e realizar um teste de similaridade florística entre outras matas ciliares espalhadas pelo Brasil, a fim de se verificar conexões florísticas entre espécies de ambientes ripários.

\section{Material e Métodos}

\section{Área de estudo}

A bacia do rio Gramame (Figura 1) está inserida em área pertencente à microrregião Litorânea Sul Paraibana, nordeste do Brasil $\left(07^{\circ} 11^{\prime} / 07^{\circ} 23^{\prime} \mathrm{S}-34^{\circ} 48^{\prime} / 35^{\circ} 10^{\prime} \mathrm{W}\right)$.

Esta bacia é estratégica para a região metropolitana da capital paraibana devido à sua contribuição para o abastecimento d'água dos municípios de João Pessoa, Bayeux, Santa Rita, Cabedelo, Pedras de Fogo e Conde, todos consistindo de centros urbanos localizados no entorno da bacia.

$\mathrm{Na}$ área de estudo, há fragmentos de mata ciliar, porém apresentando intensa interferência antrópica, tais como: residências próximas à margem, disposição

FIGURA 1: Mapa da região do rio Gramame, João Pessoa/Conde, Paraíba, nordeste do Brasil.

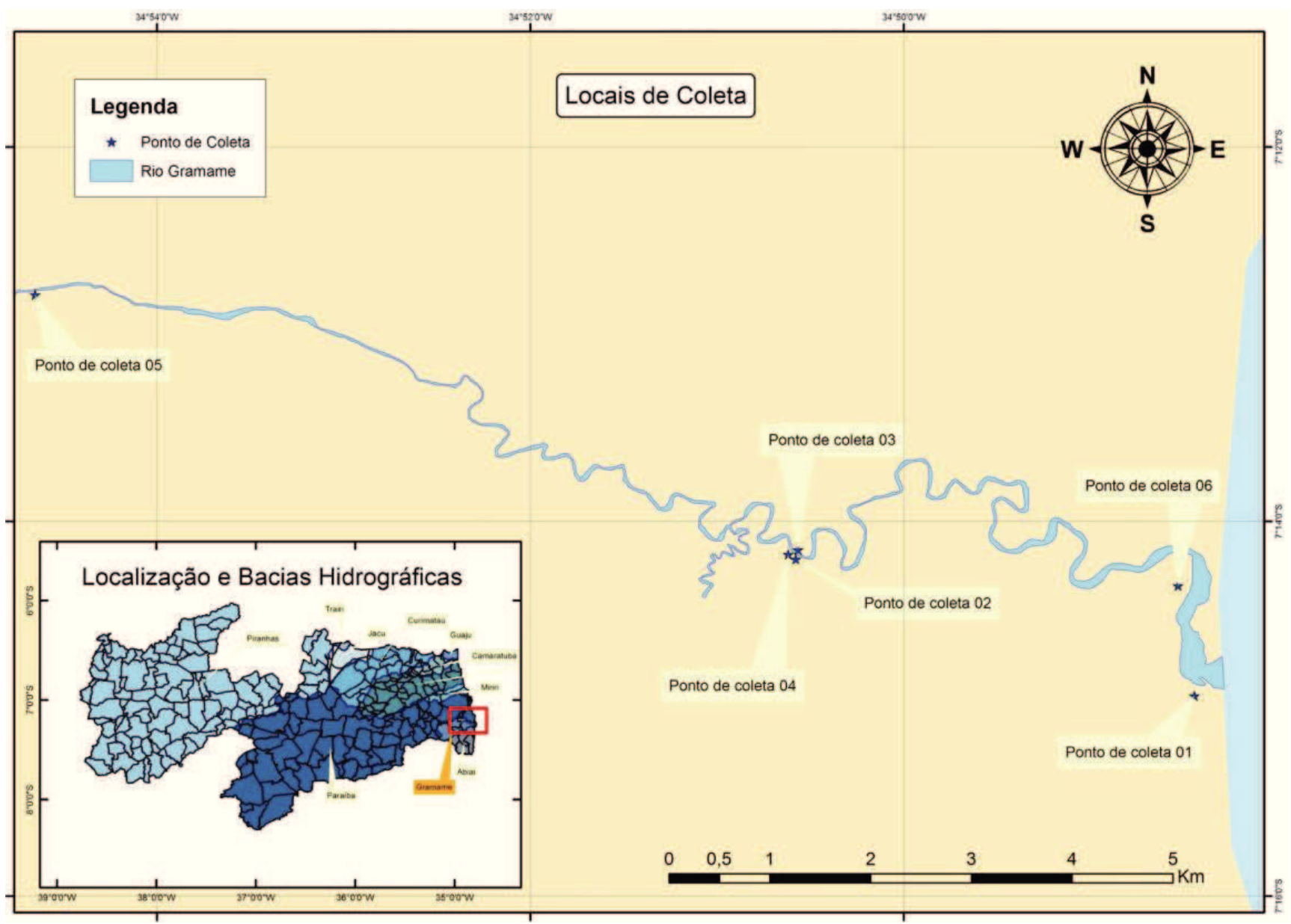


irregular de resíduos sólidos, desmatamentos, mineração de areia quartzosa, lançamento de efluentes industriais e residenciais no solo e corpo aquático, culturas agrícolas indiscriminadas, criação de animais domésticos, presença de rodovias de intenso movimento, além de ser zona de recreação e turismo em sua desembocadura.

A composição florística da mata ciliar está associada a duas condições distintas fito-fisionomicamente, uma com regime de terra firme, ou seja, nunca inundada, à montante e periodicamente inundada à jusante, pelo regime das marés.

Foram selecionados seis pontos de amostragem ao longo da bacia do rio Gramame: médio curso (BR-101), ponte situada entre os municípios de João Pessoa e Conde (rodovia PB-008) e na jusante na região de desembocadura localizada na praia de Barra de Gramame. Esses pontos foram escolhidos por apresentarem fragmentos mais "preservados" diante da situação de degradação da área.

A bacia hidrográfica do rio Gramame, apesar de ser uma das redes de drenagem mais importantes para a região do litoral central da Paraíba (PARAÍBA, 2013), tem sido uma área muito pouco estudada e com escassas informações sobre as espécies que lá ocorrem (CRIA, 2014).

\section{Composição florística e similaridade}

Os trabalhos de campo foram realizados no período de Janeiro a Outubro de 2013, a partir de caminhadas aleatórias por toda a extensão da área, visando à coleta e observação 'in loco' de material botânico (traqueófitas em estádio reprodutivo). A coleta e a herborização do material seguiram procedimentos tradicionais em estudos de plantas vasculares (BRIDSON; FORMAN, 2004).

A identificação das espécies foi fundamentada, principalmente, em artigos de cunho florístico e ou florístico-taxonômico e, também, na consulta a livros especializados como AMARAL et al. (2008) e ALVES et al. (2009). Espécimes foram ainda identificados através de comparação com amostras, preferivelmente identificadas por especialistas, nos respectivos grupos taxonômicos, depositadas nos herbários EAN e IPA.
Para evitar problemas com sinonímias, foi consultado o site The Plant List (2013) e utilizado o nome correto dos táxons.

A organização sistemática dos nomes das famílias botânicas foi feita de acordo com o APG III (2009). Os nomes das espécies e autores foram consultados na base de dados do Missouri Botanical Garden (MOBOT, 2013), The Plant List (2013) e Lista de Espécies da Flora do Brasil (FORZZA et al., 2014).

Os tipos de hábitos foram baseados em Veloso et al. (1992), complementados pelas observações feitas em campo e o espectro biológico foi baseado nas observações recomendadas por Raunkiaer (1934).

A distribuição fitogeográfica das espécies foi determinada com base em bibliografia especializada e nos sites: Global Invase Species Data Base (2013), Jstor Plant Science (2013), na base de dados do Missouri Botanical Garden (MOBOT, 2013), Flora Brasiliensis On Line (2013), Global Biodiversity Information Facility (GBIF, 2014) e Lista de Espécies da Flora do Brasil 'online' (FORZZA et al., 2014). As espécies cultivadas, tais como Anacardium occidentale, Bixa orellana, etc., não foram consideradas fitogeograficamente.

Os espécimes coletados foram incorporados ao Herbário do Instituto Agronômico de Pernambuco (IPA). Espécimes foram também incluídos na coleção do Laboratório de Biologia do Instituto Federal de Educação, Ciência e Tecnologia da Paraíba (IFPB) Campus João Pessoa.

A lista florística produzida na área do Rio Gramame foi comparada em termos de similaridade florística com a de outros inventários realizados em ambientes de vegetação ripária no Brasil: Pereira e Assis (2000), Matias e Nunes (2001), Loures et al. (2007), BaptistaMaria et al. (2008) e Scudeller e Souza (2009) situados nas regiões Norte, Nordeste, Centro-Oeste e Sudeste do Brasil, os quais adotaram metodologia semelhante à empregada no levantamento florístico do presente estudo.

A quantificação da similaridade foi avaliada com base nos índices de Jaccard (MUELLER-DOMBOIS; ELLENBERG, 1974), sendo os dados organizados em uma matriz de binária de presença/ausência das espécies 
das áreas elencadas, adotando-se o programa PAST para geração do dendrograma (HAMMER et al., 2003).

\section{Resultados}

Foram registradas 136 espécies distribuídas em 106 gêneros e 43 famílias (Tabela 1). As famílias com maior número de espécies foram: Fabaceae (19 spp.), Cyperaceae (16 spp.), Poaceae (11 spp.), e Rubiaceae (10 spp.), Asteraceae (nove spp.), Amaranthaceae e Convolvulaceae (cinco spp. cada) e Verbenaceae (quatro spp.).
Os gêneros com maior riqueza específica foram: Cyperus L. (seis spp.), Mimosa L. (cinco spp.), Borreria G. Mey. (quatro spp.), Eleocharis R. Br. (três spp.) e Alternanthera Forssk. (três spp.).

A flora da região do baixo Gramame não estava devidamente registrada até o presente momento. De um modo geral, através de informações vinculadas ao site do CRIA (2014) apenas as espécies Conocarpus erectus, Lagunaria racemosa e Rhizophora mangle foram relatadas para a área, enquanto as demais espécies não haviam sido citadas até então para a mesma.

TABELA 1: Lista de espécies registradas nos trechos estudados do rio Gramame, Paraíba, NE Brasil. Legenda Hábito: erva (E), trepadeira (T), subarbusto (SA), arbusto (A), árvore (AR), aquática (AQ); Espectro Biológico: Caméfita (Ca), Fanerófita (Fa), Geófita (Ge), Hidrófita (Hi), Hemicriptófito (Hc), Terófita (Tf); Distribuição Geográfica: Neotropical (Neo), Americana (Ame), Gondwânica (Gond), Pantropical (Pan), Cosmopolita (Cosm) e Incerta (Inc).

\begin{tabular}{|c|c|c|c|c|}
\hline Família/Espécie & Hábito & $\begin{array}{l}\text { Espectro } \\
\text { biológico }\end{array}$ & $\begin{array}{c}\text { Distribuição } \\
\text { geográfica }\end{array}$ & Voucher \\
\hline \multicolumn{5}{|l|}{ AIZOACEAE } \\
\hline Sesuvium portulacastrum (L.) L. & E & $\mathrm{Ca}$ & Pan & 88429/IPA \\
\hline \multicolumn{5}{|l|}{ AMARANTHACEAE } \\
\hline Alternanthera brasiliana (L.) Kuntze & $\mathrm{E}$ & $\mathrm{Ca}$ & Pan & 88430/IPA \\
\hline Alternanthera pungens Kunth & E & $\mathrm{Ca}$ & Pan & 018/IFPB \\
\hline Alternanthera sp. & SA & $\mathrm{Fa}$ & Inc & 065/IFPB \\
\hline Amaranthus spinosus L. & SA & $\mathrm{Ca}$ & Cosm & 041/IFPB \\
\hline Blutaparon portulacoides (A. St.-Hil.) Mears & $\mathrm{E}$ & $\mathrm{Ca}$ & Inc & 88427/IPA \\
\hline \multicolumn{5}{|l|}{ ANACARDIACEAE } \\
\hline Anacardium occidentale L. & AR & $\mathrm{Fa}$ & - & - \\
\hline Schinus terebinthifolius Raddi & $\mathrm{AR}$ & $\mathrm{Fa}$ & Pan & - \\
\hline Tapirira guianensis Aubl. & AR & $\mathrm{Fa}$ & Neo & - \\
\hline \multicolumn{5}{|l|}{ ANNONACEAE } \\
\hline Annona glabra $\mathrm{L}$. & AR & $\mathrm{Fa}$ & Pan & - \\
\hline \multicolumn{5}{|l|}{ ARACEAE } \\
\hline Caladium bicolor (Aiton) Vent. & $\mathrm{E}$ & $\mathrm{Ge}$ & Pan & - \\
\hline Montrichardia linifera (Arruda) Schott & $\mathrm{AR}$ & $\mathrm{Hi}$ & Neo & - \\
\hline \multicolumn{5}{|l|}{ ARECACEAE } \\
\hline Acrocomia intumescens Drude & $\mathrm{AR}$ & $\mathrm{Fa}$ & Neo & - \\
\hline \multicolumn{5}{|l|}{ ASTERACEAE } \\
\hline Acmella uliginosa (Sw.) Cass. & SA & $\mathrm{Hi}$ & Pan & 88480/IPA \\
\hline Aspilia pascalioides Griseb. & $\mathrm{E}$ & $\mathrm{Ca}$ & Neo & 031/IFPB \\
\hline Aspilia bonplandiana (Gardner) S.F.Blake & SA & $\mathrm{Ca}$ & Neo & 046/IFPB \\
\hline Elephantopus hirtiflorus DC. & SA & $\mathrm{Ca}$ & $\mathrm{Neo}$ & 88423/IPA \\
\hline Emilia fosbergii Nicolson & $\mathrm{E}$ & $\mathrm{Ca}$ & $\mathrm{Neo}$ & 055/IFPB \\
\hline Lourteigia ballotifolia (Kunth) R.M. King \& H. Rob. & $\mathrm{E}$ & $\mathrm{Ca}$ & $\mathrm{Neo}$ & 071/IFPB \\
\hline Tilesia baccata (L.) Pruski & $\mathrm{E}$ & $\mathrm{Ca}$ & $\mathrm{Neo}$ & 021/IFPB \\
\hline Tridax procumbens (L.) L. & $\mathrm{E}$ & $\mathrm{Ca}$ & Pan & 013/IFPB \\
\hline Indeterminada & $\mathrm{E}$ & - & Inc & 073/IFPB \\
\hline
\end{tabular}




\begin{tabular}{|c|c|c|c|c|}
\hline \multicolumn{5}{|l|}{ BIXACEAE } \\
\hline Bixa orellana L. & AR & $\mathrm{Fa}$ & - & - \\
\hline \multicolumn{5}{|l|}{ BLECHNACEAE } \\
\hline Blechnum serrulatum Rich. & A & $\mathrm{Fa}$ & Pan & - \\
\hline \multicolumn{5}{|l|}{ BORAGINACEAE } \\
\hline Heliotropium indicum L. & E & $\mathrm{Ca}$ & Cosm & 035/IFPB \\
\hline Varronia curassavica Jacq. & A & $\mathrm{Fa}$ & Neo & 88424/IPA \\
\hline \multicolumn{5}{|l|}{ CHRYSOBALANACEAE } \\
\hline Chrysobalanus icaco L. & A & Hi & Pan & 88423/IPA \\
\hline \multicolumn{5}{|l|}{ CLEOMACEAE } \\
\hline Tarenaya hassleriana (Chodat) Iltis & A & $\mathrm{Ca}$ & Cosm & 056/IFPB \\
\hline \multicolumn{5}{|l|}{ COMBRETACEAE } \\
\hline Conocarpus erectus $\mathrm{L}$. & AR & $\mathrm{Hi}$ & Gond & 011/IFPB \\
\hline Laguncularia racemosa (L.) C.F. Gaertn. & $\mathrm{AR}$ & $\mathrm{Hi}$ & Gond & 010/IFPB \\
\hline Terminalia catappa $\mathrm{L}$. & $\mathrm{AR}$ & $\mathrm{Fa}$ & Pan & - \\
\hline \multicolumn{5}{|l|}{ COMMELINACEAE } \\
\hline Commelina diffusa Burm. f. & E & $\mathrm{Ca}$ & Cosm & 052/IFPB \\
\hline \multicolumn{5}{|l|}{ CONVOLVULACEAE } \\
\hline Cuscuta racemosa Mart. & $\mathrm{T}$ & $\mathrm{Fa}$ & Neo & 031/IFPB \\
\hline Jacquemontia serrata (Choisy) Meisn. & $\mathrm{T}$ & $\mathrm{Fa}$ & Inc & 88425/IPA \\
\hline Ipomoea asarifolia (Desr.) Roem. \& Schult. & $\mathrm{T}$ & $\mathrm{Ca}$ & Gond & 040/IFPB \\
\hline Ipomoea squamosa Choisy & $\mathrm{T}$ & $\mathrm{Ca}$ & Neo & 047/IFPB \\
\hline Ipomoea vestalii Standl. & E & $\mathrm{Ca}$ & Neo & - \\
\hline Indeterminada & $\mathrm{T}$ & $\mathrm{Fa}$ & Inc & 070/IFPB \\
\hline \multicolumn{5}{|l|}{ CUCURBITACEAE } \\
\hline Citrullus lanatus (Thunb.) Matsum. \&Nakai & AR & $\mathrm{Fa}$ & Cosm & 015/IFPB \\
\hline Cucurbita sp. & $\mathrm{T}$ & $\mathrm{Fa}$ & Inc & 068/IFPB \\
\hline Momordica charantia L. & $\mathrm{T}$ & $\mathrm{Fa}$ & Pan & - \\
\hline \multicolumn{5}{|l|}{ CYPERACEAE } \\
\hline Cyperus aggregatus (Willd.) Endl. & E & $\mathrm{Hc}$ & Neo & 88468/IPA \\
\hline Cyperus diffusus Vahl & E & $\mathrm{Hc}$ & Pan & 88432/IPA \\
\hline Cyperus haspan $\mathrm{L}$. & E & $\mathrm{Hc}$ & Pan & 88438/IPA \\
\hline Cyperus ligularis $\mathrm{L}$. & $\mathrm{E}$ & $\mathrm{Hc}$ & Gond & 88457/IPA \\
\hline Cyperus luzulae (L.) Rottb. ex Retz. & E & $\mathrm{Hc}$ & Neo & 88458/IPA \\
\hline Cyperus sp. & $\mathrm{E}$ & $\mathrm{Hc}$ & Inc & 064/IFPB \\
\hline Eleocharis geniculata (L.) Roem. \& Schult. & $\mathrm{E}$ & $\mathrm{Hc}$ & Cosm & 88435/IPA \\
\hline Eleocharis glaucovirens Boeckeler & $\mathrm{E}$ & $\mathrm{Hc}$ & Neo & 88439/IPA \\
\hline Eleocharis mutata (L.) Roem. \& Schult. & $\mathrm{E}$ & $\mathrm{Hc}$ & Gond & 88442/IPA \\
\hline Fimbristylis complanata (Retz) Link & $\mathrm{E}$ & $\mathrm{Hc}$ & Cosm & - \\
\hline Fuirena umbellata Rottb. & $\mathrm{E}$ & $\mathrm{Hc}$ & Cosm & 88431/IPA \\
\hline Kyllinga odorata Vahl & $\mathrm{E}$ & $\mathrm{Hc}$ & Cosm & 029/IFPB \\
\hline Rhynchospora brevirostris Griseb. & $\mathrm{E}$ & $\mathrm{Hc}$ & Gond & 88433/IPA \\
\hline Rhynchospora rugosa (Vahl) Gale & $\mathrm{E}$ & $\mathrm{Hc}$ & Cosm & 88441/IPA \\
\hline Scleria bracteata Cav. & $\mathrm{E}$ & $\mathrm{Hc}$ & Neo & 88432/IPA \\
\hline Scleria melaleuca Reichb. ex Schldl \& Cham. & $\mathrm{E}$ & $\mathrm{Hc}$ & $\mathrm{Neo}$ & 88430/IPA \\
\hline \multicolumn{5}{|l|}{ EUPHORBIACEAE } \\
\hline Euphorbia hyssopifolia L. & $\mathrm{E}$ & $\mathrm{Tf}$ & Cosm & 88428/IPA \\
\hline Jatropha gossypiifolia L. & A & $\mathrm{Fa}$ & Pan & 048/IFPB \\
\hline Manihot sp. & A & $\mathrm{Fa}$ & Inc & - \\
\hline \multicolumn{5}{|l|}{ FABACEAE } \\
\hline Crotalaria incana $\mathrm{L}$. & SA & $\mathrm{Ca}$ & Pan & 053/IFPB \\
\hline
\end{tabular}




\begin{tabular}{|c|c|c|c|c|}
\hline Crotalaria spectabilis Roth & $\mathrm{A}$ & $\mathrm{Ca}$ & Cosm & 037/IFPB \\
\hline Centrosema brasilianum (L.) Benth. & $\mathrm{T}$ & $\mathrm{Fa}$ & Neo & 049/IFPB \\
\hline Chamaecrista flexuosa (L.) Greene & $\mathrm{E}$ & $\mathrm{Ca}$ & Neo & 050/IFPB \\
\hline Chamaecrista hispidula (Vahl) H.S.Irwin \& Barneby & $\mathrm{E}$ & $\mathrm{Ca}$ & Neo & 88420/IPA \\
\hline Clitoria laurifolia Poir. & A & $\mathrm{Fa}$ & Pan & $88417 / \mathrm{IPA}$ \\
\hline Dalbergia aff. ecastaphyllum (L.) Taub. & AR & $\mathrm{Fa}$ & Inc & 88428/IPA \\
\hline Desmodium barbatum (L.) Benth. & $\mathrm{E}$ & $\mathrm{Ca}$ & Gond & 024/IFPB \\
\hline Dioclea violacea Mart. ex Benth. & $\mathrm{T}$ & $\mathrm{Ca}$ & Neo & - \\
\hline Dioclea virgata (Rich.) Amshoff & $\mathrm{T}$ & $\mathrm{Fa}$ & $\mathrm{Neo}$ & 88419/IPA \\
\hline Indigofera hirsuta $\mathrm{L}$. & SA & $\mathrm{Ca}$ & Pan & 034/IFPB \\
\hline Macroptilium lathyroides (L.) Urb. & $\mathrm{E}$ & $\mathrm{Ca}$ & Cosm & 067/IFPB \\
\hline Mimosa caesalpinifolia Benth. & AR & $\mathrm{Fa}$ & Neo & 045/IFPB \\
\hline Mimosa quadrivalvis var. leptocarpa (DC.) Barneby & SA & $\mathrm{Ca}$ & Cosm & 88421/IPA \\
\hline Mimosa invisa Mart. ex Colla & SA & $\mathrm{Ca}$ & Pan & 025/IFPB \\
\hline Mimosa pigra $\mathrm{L}$ & SA & $\mathrm{Ca}$ & Pan & - \\
\hline Mimosa sensitiva $\mathrm{L}$. & SA & $\mathrm{Ca}$ & Neo & 88422/IPA \\
\hline Senna occidentalis (L.) Link & A & $\mathrm{Fa}$ & Cosm & 038/IFPB \\
\hline Stylosanthes scabra Vogel & SA & $\mathrm{Ca}$ & Pan & 88418/IPA \\
\hline \multicolumn{5}{|l|}{ GRATIOLACEAE } \\
\hline Scoparia dulcis L. & E & $\mathrm{Ca}$ & Ame & 030/IFPB \\
\hline \multicolumn{5}{|l|}{ HELICONIACEAE } \\
\hline Heliconia psittacorum L.f. & E & $\mathrm{Hc}$ & Neo & 012/IFPB \\
\hline \multicolumn{5}{|l|}{ LOGANIACEAE } \\
\hline Spigelia anthelmia $\mathrm{L}$. & E & $\mathrm{Ca}$ & Gond & 036/IFPB \\
\hline \multicolumn{5}{|l|}{ LYTHRACEAE } \\
\hline Cuphea flava Spreng. & A & $\mathrm{Ca}$ & Neo & 88424/IPA \\
\hline \multicolumn{5}{|l|}{ MALVACEAE } \\
\hline Apeiba tibourbou Aubl. & AR & $\mathrm{Fa}$ & $\mathrm{Neo}$ & - \\
\hline Talipariti tiliaceum var. pernanbucencis (Arruda) Fryxell. & A & $\mathrm{Hi}$ & $\mathrm{Neo}$ & 028/IFPB \\
\hline Pavonia cancellata $(\mathrm{L}$.$) Cav.$ & $\mathrm{E}$ & $\mathrm{Ca}$ & $\mathrm{Neo}$ & 033/IFPB \\
\hline Sidastrum micranthum (A. St.-Hil.) Fryxell & A & $\mathrm{Ca}$ & Neo & 060/IFPB \\
\hline Urena lobata $\mathrm{L}$. & $\mathrm{E}$ & $\mathrm{Ca}$ & Cosm & 88482/IPA \\
\hline Urena sp. & $\mathrm{E}$ & $\mathrm{Ca}$ & Inc & 069/IFPB \\
\hline \multicolumn{5}{|l|}{ MELASTOMATACEAE } \\
\hline Acisanthera variabilis (DC.) Triana & $\mathrm{E}$ & $\mathrm{Ca}$ & $\mathrm{Neo}$ & 017/IFPB \\
\hline Nepsera aquatica (Aubl.) Naudin & SA & $\mathrm{Ca}$ & Neo & 88426/IPA \\
\hline Pterolepis glomerata (Rottb.) Miq. & $\mathrm{E}$ & $\mathrm{Ca}$ & $\mathrm{Neo}$ & 019/IFPB \\
\hline \multicolumn{5}{|l|}{ MYRTACEAE } \\
\hline Syzygium cumini (L.) Skeels & $\mathrm{AR}$ & $\mathrm{Fa}$ & Pan & - \\
\hline \multicolumn{5}{|l|}{ OCHNACEAE } \\
\hline Sauvagesia erecta $\mathrm{L}$. & SA & $\mathrm{Ca}$ & Gond & 88425/IPA \\
\hline \multicolumn{5}{|l|}{ ONAGRACEAE } \\
\hline Ludwigia erecta (L.) H. Hara & $\mathrm{E}$ & $\mathrm{Hi}$ & Gond & 026/IFPB \\
\hline Ludwigia octovalvis (Jacq.) P.H. Raven & SA & $\mathrm{Hi}$ & Cosm & 88429/IPA \\
\hline \multicolumn{5}{|l|}{ PIPERACEAE } \\
\hline Piper marginatum Jacq. & A & $\mathrm{Ca}$ & Neo & 061/IFPB \\
\hline \multicolumn{5}{|l|}{ POACEAE } \\
\hline Cenchrus echinatus $\mathrm{L}$. & $\mathrm{E}$ & $\mathrm{Tf}$ & Neo & 001/IFPB \\
\hline Chloris barbata $\mathrm{Sw}$. & $\mathrm{E}$ & $\mathrm{Ca}$ & Cosm & 004/IFPB \\
\hline Dactyloctenium aegyptium (L.) Willd. & E & $\mathrm{Ca}$ & Cosm & 006/IFPB \\
\hline Digitaria insularis (L.) Fedde & $\mathrm{E}$ & $\mathrm{Ca}$ & $\mathrm{Neo}$ & 007/IFPB \\
\hline
\end{tabular}




\begin{tabular}{|c|c|c|c|c|}
\hline Eleusine indica (L.) Gaertn. & $\mathrm{E}$ & Tf & Cosm & 043/IFPB \\
\hline Eragrostis ciliaris (L.) R.Br. & $\mathrm{E}$ & $\mathrm{Ca}$ & Gond & 008/IFPB \\
\hline Guadua aff. paraguayana Döll & AR & $\mathrm{Fa}$ & Neo & - \\
\hline Melinis repens (Willd.) Zizka & $\mathrm{E}$ & $\mathrm{Ca}$ & Pan & 009/IFPB \\
\hline Paspalum vaginatum $\mathrm{Sw}$. & $\mathrm{E}$ & $\mathrm{Hc}$ & Cosm & - \\
\hline Paspalum millegrana Schrad. & $\mathrm{E}$ & $\mathrm{Hc}$ & Neo & - \\
\hline Indeterminada & $\mathrm{E}$ & - & Inc & - \\
\hline \multicolumn{5}{|l|}{ PTERIDACEAE } \\
\hline Acrostichum danaeifolium Langsd. \& Fisch. & A & Hi & Neo & - \\
\hline \multicolumn{5}{|l|}{ RHIZOPHORACEAE } \\
\hline Rhizophora mangle L. & AR & Hi & Gond & 030/IFPB \\
\hline \multicolumn{5}{|l|}{ RUBIACEAE } \\
\hline Borreria capitata (Ruiz \& Pav.) DC. & $\mathrm{E}$ & $\mathrm{Ca}$ & Neo & $88415 /$ IPA \\
\hline $\begin{array}{l}\text { Borreria ocymifolia (Willd. ex Roem. \& Schult.) Bacigalupo } \\
\text { \& E.L. Cabral }\end{array}$ & SA & $\mathrm{Ca}$ & Neo & 88412/IPA \\
\hline Borreria sp. & SA & $\mathrm{Ca}$ & Inc & 066/IFPB \\
\hline Diodella apiculata (Willd.) Delprete & $\mathrm{E}$ & $\mathrm{Ca}$ & $\mathrm{Neo}$ & 88407/IPA \\
\hline Diodia teres Walter & $\mathrm{E}$ & $\mathrm{Tf}$ & Cosm & 88411/IPA \\
\hline Mitracarpus frigidus (Willd. ex Roem. \& Schult.) K. Schum. & SA & $\mathrm{Ca}$ & Neo & 88413/IPA \\
\hline Sabicea grisea Cham. \& Schltdl. & A & $\mathrm{Fa}$ & Neo & 88414/IPA \\
\hline Spermacoce verticillata $\mathrm{L}$. & $\mathrm{E}$ & $\mathrm{Ca}$ & Pan & 88416/IPA \\
\hline Richardia brasiliensis Gomes & $\mathrm{E}$ & $\mathrm{Ca}$ & Cosm & 88408/IPA \\
\hline Richardia grandiflora (Cham. \& Schult.) Steud. & $\mathrm{E}$ & $\mathrm{Tf}$ & Neo & 88410/IPA \\
\hline \multicolumn{5}{|l|}{ RUTACEAE } \\
\hline Poncirus sp. & AR & $\mathrm{Fa}$ & Inc & - \\
\hline \multicolumn{5}{|l|}{ SAPINDACEAE } \\
\hline Paullinia pinnata $\mathrm{L}$. & $\mathrm{T}$ & $\mathrm{Fa}$ & Gond & 88427/IPA \\
\hline \multicolumn{5}{|l|}{ SOLANACEAE } \\
\hline Indeterminada & SA & $\mathrm{Ca}$ & Inc & - \\
\hline \multicolumn{5}{|l|}{ TURNERACEAE } \\
\hline Turnera melochioides Cambess. & SA & $\mathrm{Ca}$ & $\mathrm{Neo}$ & 044/IFPB \\
\hline Turnera subulata $\mathrm{Sm}$. & SA & $\mathrm{Ca}$ & Neo & 014/IFPB \\
\hline \multicolumn{5}{|l|}{ URTICACEAE } \\
\hline Cecropia pachystachya Trécul. & AR & $\mathrm{Fa}$ & $\mathrm{Neo}$ & 023/IFPB \\
\hline Pilea microphylla (L.) Liebm. & $\mathrm{E}$ & $\mathrm{Ca}$ & Cosm & - \\
\hline Urtica dioica $\mathrm{L}$. & $\mathrm{E}$ & $\mathrm{Ca}$ & Cosm & - \\
\hline \multicolumn{5}{|l|}{ VERBENACEAE } \\
\hline Lantana camara $\mathrm{L}$. & $\mathrm{E}$ & $\mathrm{Fa}$ & $\mathrm{Neo}$ & 022/IFPB \\
\hline Lantana radula $\mathrm{Sw}$ & SA & $\mathrm{Fa}$ & $\mathrm{Neo}$ & 88481/IPA \\
\hline Lippia alba (Mill.) N. E. Br. ex Britton \& P. Wilson & SA & $\mathrm{Ca}$ & Neo & 039/IFPB \\
\hline Stachytarpheta elatior Schrad. ex Schult. & $\mathrm{E}$ & $\mathrm{Ca}$ & $\mathrm{Neo}$ & 019/IFPB \\
\hline \multicolumn{5}{|l|}{ XYRIDACEAE } \\
\hline Xyris jupicai Rich. & $\mathrm{E}$ & $\mathrm{Hc}$ & $\mathrm{Neo}$ & 027/IFPB \\
\hline \multicolumn{5}{|l|}{ ZYGOPHYLLACEAE } \\
\hline Kallstroemia maxima (L.) Hook. \& Arn. & $\mathrm{E}$ & $\mathrm{Ca}$ & $\mathrm{Neo}$ & - \\
\hline
\end{tabular}


Aárea de estudo, está naturalmente compartimentada em mata de montante e mata de jusante. Essas duas formações situadas ao longo do curso d'água constituem formação florestal de terra firme (montante) e planície inundável pelo regime de marés (jusante), respectivamente. Houve um maior número de espécies na mata na região da montante (aproximadamente 85\% das espécies) e uma menor quantidade de espécies à jusante, haja vista que em se tratando de uma planície inundável, há uma restrição maior sobre a tolerância, de determinados táxons, nesses bosques inundáveis, pela hipoxia do solo (ALVARADO, 2008).

$\mathrm{Na}$ região de mata inundável, prevaleceram basicamente as espécies de Anacardiaceae (Anacardium occidentale, Schinus terebinthifolius, Tapirira guianensis), Aizoaceae (Sesuvium portulacastrum), Combretaceae (Conocarpus erectus, Laguncularia racemosa, Terminalia catappa), Cucurbitaceae (Citrullus lanatus), Cuscutaceae (Cuscuta racemosa) e Rhizophoraceae (Rhizophora mangle), podendo-se evidenciar a prevalência desses táxons em relação a condição de alta salinidade do solo e na região de manguezal, anoxia do solo. As demais espécies estiveram relacionadas às áreas coletadas em mata de terra firme.

Em relação aos tipos de hábito encontrados na área de estudo (Figura 2), predominou o herbáceo, seguido dos hábitos arbóreo, o subarbustivo, o arbustivo e o trepador. $\mathrm{O}$ elevado percentual apresentado pelo componente herbáceo indica, especialmente, a partir das observações de campo, que está relacionado à descaracterização da área de estudo, por atividades antrópicas, pela supressão da vegetação de grande porte. Evidenciou-se que a vegetação de grande porte, se restringiu a borda do rio ou na área de manguezal.

Em relação ao espectro biológico (Figura 3), obtiveram-se as formas de vida caméfita predominantemente, seguida das formas fanerófita, hemicriptófita, hidrófita, terófita e geófita. Os organismos caméfitos e hemicriptófitos são encontrados em um expressivo trecho da área de estudo, também associados aos antropizados e de borda. Nas áreas próximas às margens do rio Gramame são encontrados fanerófitos, onde ocorre naturalmente a mata ciliar estrita, com presença de árvores de médio e grande porte, formando um dossel definido.
FIGURA 2: Riqueza de espécies encontradas, em relação ao seu habito, nos trechos analisados da mata ciliar do rio Gramame, Paraíba.

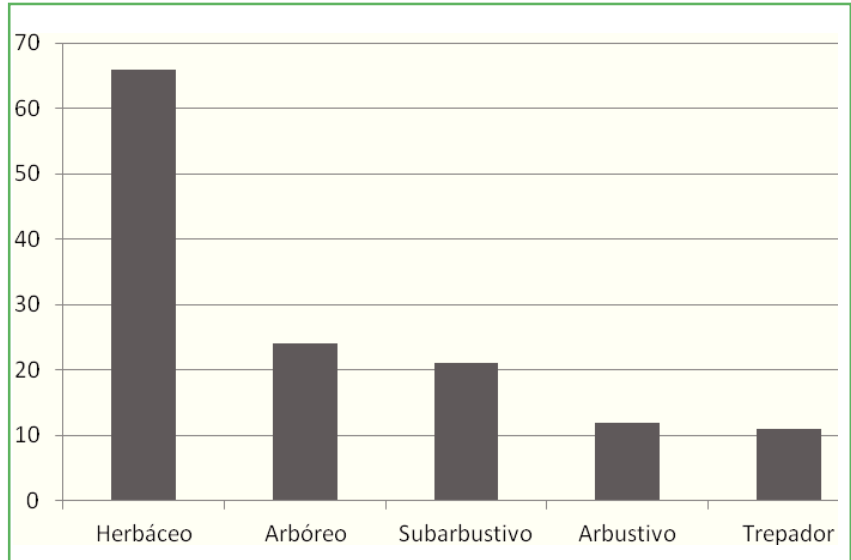

FIGURA 3: Espectro biológico (segundo RAUNKIAER, 1934) das espécies registradas nos trechos analisados da mata ciliar do rio Gramame, Paraíba.

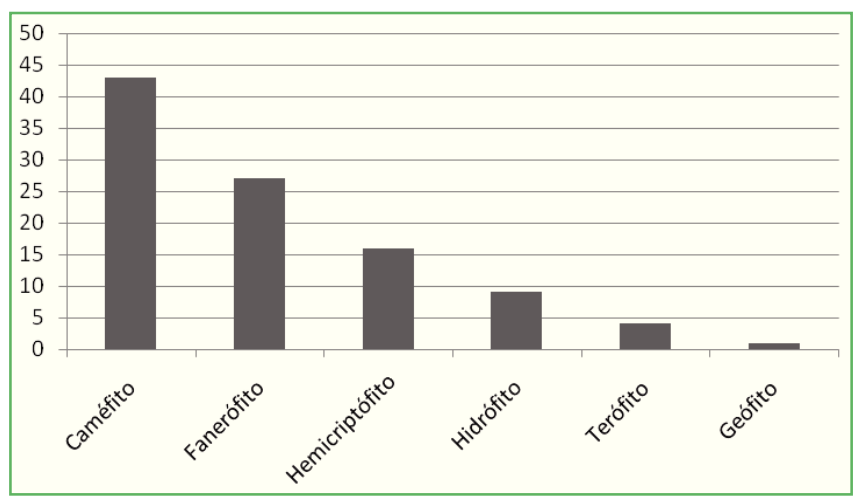

Os padrões de distribuição geográfica (Figura 4) evidenciaram que as espécies que ocorrem estritamente na província Neotropical estiveram representadas em maior número. Em seguida, destacam-se as espécies com ampla ocorrência para a região Pantropical, espécies de domínio Cosmopolita, Gondwânicas e de distribuição exclusivamente Americana. No entanto, a composição florística está composta basicamente por espécies amplamente distribuídas.

As espécies indeterminadas ao nível específico ou que apresentaram identificação incerta (Alternanthera sp., Borreria sp., Cucurbita sp., Dalbergia aff. ecastaphyllum, Cyperus sp., Guadua aff. paraguayana, Manihot sp., Poncirus sp. e Urena sp.) não puderam ter os padrões de distribuição geográfica confirmados. 
FIGURA 4: Padrões de distribuição geográfica das espécies encontradas nos trechos analisados da mata ciliar do rio Gramame, Paraíba.

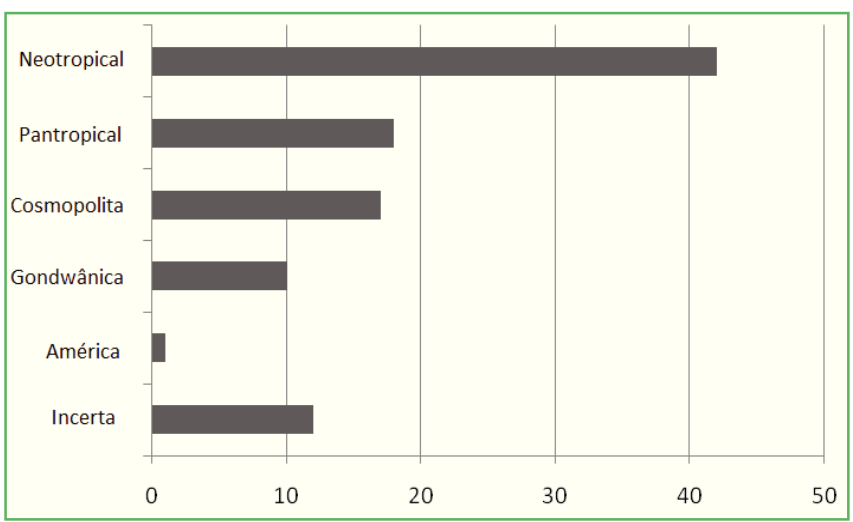

Em relação à análise de similaridade entre os ecossistemas comparados neste estudo, evidenciou-se um coeficiente cofenético de 97,25\%, destacando uma maior similaridade entre as áreas enquadradas como costeiras (Figura 5).

FIGURA 5: Dendrograma da análise de agrupamento de áreas comparadas de mata ciliar do Brasil, com base no coeficiente de similaridade de Jaccard. Legenda: A (Este estudo); B (PEREIRA; ASSIS, 2000); C (LOURES et al., 2007); D (MATIAS; NUNES, 2001); E (BAPTISTA-MARIA et al., 2008); F (SCUDELLER; SOUZA, 2009).

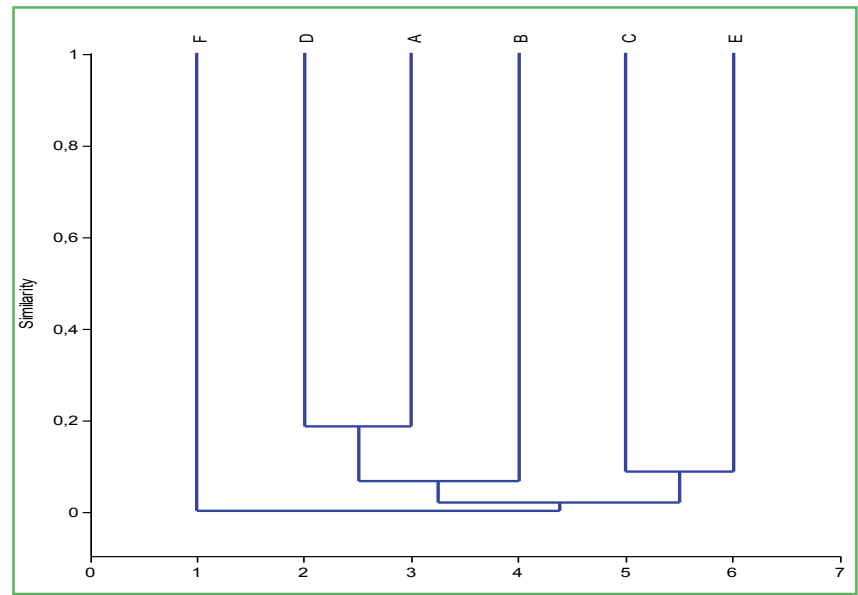

\section{Discussão}

Em relação à composição florística evidenciada e baseando-se na consulta de outros estudos florísticos (e.g., PEREIRA; ASSIS, 2000; LACERDA et al., 2005; 2007; ANDRADE et al., 2006; BAPTISTA-MARIA et al., 2009; RIBEIRO-FILHO et al., 2009; SCUDELLER;
SOUZA, 2009; SOUZA et al., 2011), verifica-se que, predominantemente, Fabaceae é o táxon mais bem representado nos principais ecossistemas ribeirinhos brasileiros (Restinga, Floresta Atlântica, Cerrado, Caatinga, Amazônia).

Em termos de riqueza específica das subfamílias de Fabaceae foram registrados os seguintes resultados: Papilionoideae, com 56\%; Mimosoideae, com 27,5\%, e Caesalpinioideae, com 16,5\% do total de espécies da família na área estudada.

Segundo SPRENT (1995), a prevalência de Fabaceae - subfamília Papilionoideae, como também registrado neste trabalho, constitui um grupo mais nodulante e, por fim, com potencial maior de fixação de nitrogênio para si e potencial disponibilização para o meio. Nesse contexto, Allen e Allen (1981) destacam que, Papilionoideae, por formar um grupo mais sensível na fixação de nitrogênio, seriam também espécies mais susceptíveis, em vantagens ecológicas ao se estabelecerem nessas condições. Sweeney et al. (2004) confirmam que essas áreas ripárias proporcionam potencialmente maior quantidade de fixação de nitrogênio, daí a importância desse componente florístico nessas áreas ribeirinhas.

Ainda sobre a composição florística da área, outra família também comumente registrada em áreas ripárias é Euphorbiaceae (PEREIRA; ASSIS, 2000; LOURES et al., 2007; BAPTISTA-MARIA et al., 2009), Rubiaceae (ALVARADO, 2008), além de Cyperaceae e Poaceae (ZEPEDA-GÓMEZ et al., 2012), estas últimas consideradas componentes significativos, principalmente em regiões praieiras onde há fortes pressões ambientais típicas de zonas litorâneas (insolação, ventos constantes, solos salinos, dentre outros) (MATIAS; NUNES, 2001). Essa observação corrobora com os dados observados neste trabalho, em que se constatou a predominância de espécies de Poaceae e Cyperaceae, nas zonas mais abertas e ensolaradas.

Com relação ao espectro biológico das espécies inventariadas, a predominância de vegetais caméfitos dáse pelo fato dessa vegetação abarcar espécies de hábito herbáceo estolonífero e rizomatoso, estratégias que muito bem se adaptam às condições ambientais próprias 
de áreas de restingas. Essa forma de vida também é dominante em áreas de solos rasos ou pedregosos, como na fitofisionomia do tipo estepe (MIODUSKI; MORO, 2011).

Em ambientes de borda, há ocorrência também de vegetais hemicriptófitos, indicando a existência de uma vegetação mais espaçada, onde a incidência de luz no solo é maior. Essa forma de vida está relacionada a "mecanismos" biológicos, que facilitam o seu desenvolvimento em áreas mais abertas e secas (COSTA et al., 2007). Em regiões de elevadas altitudes, onde também o vento e escassez hídrica no solo são fatores limitantes, os hemicriptófitos também são dominantes (MONTESINOS-TUBÉE, 2011).

Observando-se também nas áreas de focos de antropização, em vários trechos da área estudada, o surgimento de hemi-criptófitas, pela exposição constante dos vegetais à incidência direta da luz solar e selecionando vegetais mais adaptados ao solo com baixa retenção hídrica, onde Cyperaceae apresentaram expressivas populações. A literatura confirma a situação observada, em constatações de áreas de solos pobres e secos (ZIPPARRO et al., 2005).

Já o componente fanerofítico se restringe às zonas marginais do rio. Nesse sentido, Adjakpa et al. (2013) observam a prevalência também do grupo de fanerófitos na vegetação componente à margem de um rio africano em condições climáticas similares e Almeida-Júnior e Zickel (2009) confirmam a observação feita durante estudo ao inventariarem espécies fanerófitas em uma área de restinga do nordeste do Brasil, cuja preferência de microhábitat é também a margem do rio.

No que se referem à distribuição geográfica das espécies pesquisadas, apresentaram ampla distribuição geográfica. Esse aspecto provavelmente indica que a área de estudo, por sofrer pressão de ordem antrópica (desmatamento indiscriminado, principalmente), ocasionou a instalação de uma flora generalista (resistente a variações climáticas e perturbações ambientais, por exemplo) e com estratégias biológicas de dispersão apropriadas a estes casos. Alguns autores (PIŠEK et al., 2004; SHAN-HUAH et al., 2009; HASSEMER; TREVISAN, 2012) evidenciam que as floras de ampla distribuição geográfica estão relacionadas ao fato de se instalarem em ambientes tipicamente degradados.

Quando se fala em ecossistemas degradados, logo se pode remeter ao caso da história ambiental dos ecossistemas costeiros. Essas regiões litorâneas, onde foi conduzido o presente estudo, sofreram uma urbanização (acompanhada de degradação) histórica não só no Brasil (SUHOGUSOFE; PILIACKAS, 2007), como também na Europa (DUFOUR; PIÉGAY, 2006), por exemplo.

Em relação à análise de similaridade, a área estudada por Scudeller e Souza (2009) [F] foi a que apresentou maior distância em termos de similaridade florística. Os autores inventariaram uma mata de igapó na Amazônia central, com condições climáticas equatoriais. A única espécie em comum, entre todos os trabalhos incluídos na análise, foi Psidium guineense (Myrtaceae), que também foi citada no estudo de Pereira e Assis (2000).

Em seguida, as floras assentadas no Brasil central, estudadas por Loures et al. (2007) [C] e Baptista-Maria et al. (2008) [E]. No primeiro [C], foi inventariado um fragmento de floresta paludosa de Mata Atlântica no Alto do Rio Pardo, Sul de Minas Gerais, apresentando clima subtropical úmido. O segundo [E] trata de um estudo florístico realizado em uma mata ciliar associada ao rio Formoso, Bonito e Mato Grosso do Sul, a região possui o clima tropical chuvoso de Savana e está situada no domínio do Cerrado. Essas duas floras compartilharam vários táxons, e as famílias mais representativas foram Myrtaceae, Lauraceae e Euphorbiaceae.

A área estudada por Pereira e Assis (2000) [B] apresentou-se floristicamente conectada às áreas estudadas por Matias e Nunes (2001) [D] e com a do presente estudo [A]. Tais áreas encontram-se assentadas em restingas, porém em zonas geograficamente distintas entre si (estados do Espírito Santo, Ceará e Paraíba, respectivamente).

O estudo [B] consiste no levantamento florístico de uma reserva ecológica costeira no sudeste brasileiro (Camburi, Vitória), apresentando um clima tropical quente-úmido. Em comum, foram registradas espécies das famílias Anacardiaceae (Anacardium occidentale, Schinus terebinthifolius, Tapirira guianensis), Fabaceae 
(Stylosanthes scabra), Boraginaceae (Heliotropium indicum), Ochnaceae (Sauvagesia erecta), Rubiaceae (Borreria verticillata), Cyperaceae (Cyperus ligularis, Fuirena umbellata), Xyridaceae (Xyris jupicai) e Blechnaceae (Blechnum serrulatum), todas elas de ampla distribuição geográfica (GBIF, 2014), com apenas uma espécie (Cuphea flava), pertencente à família Lythraceae, endêmica da flora brasileira.

O estudo [D] apresenta o levantamento florístico em uma Área de Proteção Ambiental (APA) em Jericoacoara, Ceará, apresentando também campos dunários, estando instalada em zona climática do tipo quente e úmida (Aw'sg. Köppen). Compartilham com a área de Gramame (Paraíba), [A] espécies de Aizoaceae (Sesuvium portulacastrum), Amaranthaceae (Alternanthera brasiliana), Anacardiaceae (Anacardium occidentale), Chrysobalanaceae (Chrysobalanus icaco), Combretaceae (Conocarpus erectus, Langucularia racemosa), Convolvulaceae (Ipomoea asarifolia), Cyperaceae (Cyperus ligularis, Eleocharis geniculata), Malvaceae (Pavonia cancellata), Turneraceae (Turnera subulata), Rhizophoraceae (Rhizophora mangle), Rubiaceae (Borreria verticillata, Diodia teres, Mitracarpus frigidus, Richardia grandiflora) e Poaceae (Cenchrus echinatus, Paspalum vaginatum).

Evidenciou-se, que a similaridade florística esteve mais relacionada às condições ambientais de hábitat costeiros (tropical sempre úmido), onde espécies menos fastigiosas quanto ao tipo de solo, suportando ambiente ensolarado, com forte massas de ventos, além dos fatores de antropização, estiveram mais relacionadas entre si.

Diante do exposto, este estudo destacou a composição florística, tipos de hábitos, espectro biológico e os padrões de distribuição geográfica e relacionou a similaridade de uma área de mata ciliar impactada. Boa parte das áreas analisadas (PEREIRA; ASSIS, 2000; MATIAS; NUNES, 2001; LOURES et al., 2007; SOUZA et al., 2001), estão assentadas em perímetros urbanos, o que representam problemas crônicos, já supracitados, nos ecossistemas ciliares do Brasil. Esses estudos denunciam esses problemas de degradação ambiental, o que são igualmente observados ao longo da bacia do rio Gramame, Paraíba.
O valor ambiental sobre as informações fornecidas por essas Check Lists representam principalmente um alerta ambiental sobre floras que vêm sofrendo pressão antropogênica progressiva pelas atividades urbanísticas locais e total desrespeito à legislação vigente.

Esses resultados revelam-se como parâmetro para análise de estudos de impactos ambientais sobre essas floras e reforçando a necessidade da preservação dos ecossistemas ripários. Por essa razão, impõem-se ações prioritárias de fiscalização baseadas em uma legislação que, efetivamente, proteja a mata ciliar, a fim de reprimir a exploração indiscriminada das florestas ribeirinhas.

\section{Agradecimentos}

Ao CNPq, pela concessão de bolsa PIBIC-EM a T. S. Farias e M. V. Santos (Processo: 23381.004234.201236/PRPIPG-IFPB); J. I. M. Melo ao CNPq a Bolsa de Produtividade em Pesquisa (PQ-2); ao IFPB, pela concessão da bolsa institucional de pesquisador e taxa de bancada a H. O. Machado-Filho (23326.009791.201216/IFPB-JP); ao Dr. Sergio Romero da Silva Xavier pela confirmação dos nomes das "pterifóditas" coletadas; à curadora do Herbário Dárdano de Andrade-Lima, Instituto Agronômico de Pernambuco (IPA), Drª ${ }^{\mathrm{a}}$. Rita de Cássia Araújo Pereira, pelas confirmações/identificações de espécies de angiospermas deste estudo, junto com seus colaboradores Ana Luíza Du Bocage Neta, Fernando Antônio Távora Galindo e Maria Olivia de Oliveira Cano.

\section{Referências}

ADJAKPA, J. B.; YEDOMONHAN, H.; AHOTON, L. E.; WEESIE, P. D. M.; AKPO, L. E. Structure et diversité floristique des îlots de forêts riveraines communautaires de la Basse vallée de la Sô au Sud-Est du Bénin. Journal of Applied Biosciences, Grahamstown, v. 65, n. 1, p. 4902-4911, 2013.

ALLEN, O. N.; ALLEN, E. K. The Leguminosae: a source book of characteristics use and nodulation. Wisconsin: University of Wisconsin Press, 1981. 812 p.

ALMEIDA-JÚNIOR, J. R.; ZICKEL, C. S. Fisionomia psamófilareptante: riqueza e composição de espécies na praia da Pipa, Rio Grande do Norte, Brasil. Pesquisas, Botânica, São Leopoldo, v. 1, p. 289-299, 2009

ALVARADO, H. Aspectos estruturales y florísticos de cuatro bosques ribeños de la cuenca del río Aroa, Estado Yaracuy, 
Venezuela. Acta Botánica Venezuelana, Caracas, v. 31, n. 1, p. 273-290, 2008.

ALVES, M.; ARAÚJO, M. F.; MACIEL, J. R.; MARTINS, S. Flora de Mirandiba. Recife: Associação Plantas do Nordeste, 2009. 357 p.

AMARAL, M. C. E.; BITTRICH, V.; FARIA, A. D.; ANDERSON, L. O.; AONA, L. Y. S. Guia de campo para plantas aquáticas e palustres do estado de São Paulo. Ribeirão Preto: Holos, 2008. $452 \mathrm{p}$.

ANDRADE, L. A.; OLIVEIRA, F. X.; NASCIMENTO, I. S.; FABRICANTE, J. R.; SAMPAIO, E. V. S. B.; BARBOSA, M. R. V. Análise florística e estrutural de matas ciliares ocorrentes em brejo de altitude no município de Areia, Paraíba. Revista Brasileira de Ciências Agrárias, Recife, v. 1, n. 1, p. 31-40, 2006.

APG III. An update of the Angiosperm Phylogeny Group classification for orders and families of flowering plants: APG III. Botanical Journal of the Linnean Society, London, v. 161, n. 1, p. 105-121, 2009.

BAPTISTA-MARIA, V. R.; RODRIGUES, R. R.; DAMASCENOJÚNIOR, G.; MARIA, F. S.; SOUZA, V. C. Composição florística de florestas estacionais ribeirinhas no estado de Mato Grosso do Sul, Brasil. Acta Botanica Brasilica, Feira de Santana, v. 23, n. 2, p. $535-548,2009$.

BRASIL. Lei $\mathbf{n}^{\mathbf{0}} \mathbf{7 . 8 0 3}$, de 18 de julho de 1989. Altera a redação da Lei $n^{\circ} 4.771$, de 15 de setembro de 1965 , e revoga as Leis $n^{\circ} \mathrm{s}$ 6.535, de 15 de junho de 1978, e 7.511, de 7 de julho de 1986 . Brasília, 1989.

BRIDSON, D.; FORMAN, L. The herbarium handbook. Kew: The Royal Botanic Garden, 2004. 346 p.

CBD - CONVENTION ON BIOLOGICAL DIVERSITY. Identificação, monitorização, indicadores e avaliações. 2013. Disponível em <http://www.cbd.int/>. Acesso em: 8 fevereiro 2013. COSTA, R. C.; ARAÚJO, F. S.; LIMA-VERDE, L. W. Flora and life-form spectrum in an area of deciduous thorn woodland (caatinga) in northeastern, Brasil. Journal of Arid Environments, London, v. 68, n. 1, p. 237-247, 2007.

CRIA. Centro de Referência em Informação Ambiental. 2014. Disponível em <http://www.cria.org.br>. Acesso em 12 abril 2014. DUFOUR, S.; PIÉGAY, P. Forêts riveraines des cours d'eau et ripisylves: spécificités, fonctions et gestion. Revue Forestière Française, Nancy, v. 58, n. 1, p. 339-350, 2006.

ESTEVES, F. A. Fundamentos de Limnologia. 3 ed. Rio de Janeiro: Interciência, 2011. 790 p.

FAO - FOOD AND AGRICULTURE ORGANIZATION OF THE UNITED NATIONS. Global forest resources assessment FRA. Main Report. FAO Forestry Papers, 163. Roma: FAO, 2010. 378 p.

FLORA BRASILIENSIS ON LINE. Flora brasiliensis on line.
2013. Disponível em <http://florabrasiliensis.cria.org.br/>. Acesso em: 12 outubro 2013.

FORZZA, R. C.; LEITMAN, P. M.; COSTA, A. F.; CARVALHO JR., A. A.; PEIXOTO, A. L.; WALTER, B. M. T.; BICUDO, C.; ZAPPI, D.; COSTA, D. P.; LLERAS, E.; MARTINELLI, G.; LIMA, H. C.; PRADO, J.; STEHMANN, J. R.; BAUMGRATZ, J. F. A.; PIRANI, J. R.; SYLVESTRE, L.; MAIA, L. C.; LOHMANN, L. G.; QUEIROZ, L. P.; SILVEIRA, M.; COELHO, M. N.; MAMEDE, M. C.; BASTOS, M. N. C.; MORIM, M. P.; BARBOSA, M. R.; MENEZES, M.; HOPKINS, M.; SECCO, R.; CAVALCANTI, T. B.; SOUZA, V. C. Lista de espécies da flora do Brasil. 2014. Disponível em <http://floradobrasil.jbrj.gov.br/jabot/listaBrasil/>. Acesso em: 14 agosto 2014.

GBIF - GLOBAL BIODIVERSITY INFORMATION FACILITY. Occurrence overview. 2013. Disponível em $<$ http:// www.gbif. org/>. Acesso em: 12 janeiro 2014.

GIBBS, P. E.; LEITÃO-FILHO, H. F.; ABBOT, R. J. Application of the point-centred quarter method in a floristic survey of a area of Gallery Forest at Moji-guaçu, SP, Brasil. Revista Brasileira de Botânica, São Paulo, v. 3, n. 1, p. 17-22, 1980.

GLOBAL INVASIVE SPECIES DATABASE. Global invasive species database. 2013. Disponível em: <http://www.issg.org/ database/>. Acesso em: 3 set. 2013.

HAMMER, O.; HARPER, D. A. T.; RYAN, P. D. Paleontological statistics - PAST. Version 1.18. 2003. Disponível em < http://www. folk.uio.no/ohammer/past>. Acesso em: 28 out. 2003.

HASSEMER, G.; TREVISAN, R. Levantamento florístico de plantas vasculares espontâneas em ambientes antrópicos no campus da Universidade Federal de Santa Catarina, Florianópolis, Brasil. Biotemas, Florianópolis, v. 25, n. 3, p. 75-96, 2012.

JSTOR PLANT SCIENCE. Jstor Plant Science. 2013. Disponível em: <http://plants.jstor.org>. Acesso em: 3 set. 2013.

KAGEYAMA, P. Y.; GANDARA, F. B.; OLIVEIRA, R. E.; MORAES, L. F. Restauração da mata ciliar - manual para recuperação de áreas ciliares e microbacias. Rio de Janeiro: Semads, 2001. 104 p.

KILCA, R. V.; JARENKOW, J. A.; SOARES, J. C. W.; GARCIA, E. N. Florística e fitofisionomias da planície de inundação do rio Piratini e a sua importância para conservação no Pampa do Rio Grande do Sul, Brasil. Neotropical Biology and Conservation, São Leopoldo, v. 6, n. 3, p. 227-249, 2011.

LACERDA, A. V.; BARBOSA, F. M.; BARBOSA, M. R. V. Estudo do componente arbustivo-arbóreo de matas ciliares na bacia do rio Taperoá, semi-árido Paraibano: uma perspectiva para a sustentabilidade dos recursos naturais. Oecologia Brasiliensis, Rio de Janeiro, v. 11, n. 3, p. 331-340, 2007.

LACERDA, A. V.; NORDI, N.; BARBOSA, F. M.; WATANABE, T. Levantamento florístico do componente arbustivo-arbóreo da vegetação ciliar do rio Taperoá, PB, Brasil Acta Botanica Brasilica, São Paulo, v. 19, n. 3, p. 647-656, 2005.

LEANDRO, M. D.; VIVEIROS, C. A. F. Mata ciliar, área de preservação permanente. Linha Direta, Belo Horizonte, v. 1, n. 296, p. 1, 2003.

LOURES, L.; CARVALHO, D. A.; MACHADO, E. L. M.; MARQUES, J. J. G. S. M. Florística, estrutura e características do solo de um fragmento de floresta paludosa no sudeste do Brasil. 
Acta Botanica Brasilica, São Paulo, v. 21, n. 4, p. 885-896, 2007.

MARCHI, T. C.; JARENKOW, J. A. Estrutura do componente arbóreo de mata ribeirinha do rio Camaquã, município de Cristal, Rio Grande do Sul, Brasil. Iheringia, Botânica, Porto Alegre, v. 63, n. 2, p. 241-248, 2008.

MARQUES, M.; SILVA, S.; SALINO, A. Florística e estrutura do componente arbustivo-arbóreo de uma floresta higrófila da bacia do río Jacaré Pepira, SP, Brasil. Acta Botanica Brasilica, São Paulo, v. 17, n. 4, p. 495-506, 2003.

MATIAS, L. Q.; NUNES, E. P. Levantamento florístico da área de proteção ambiental de Jericoacoara, Ceará. Acta Botanica Brasilica, Porto Alegre, v. 15, n. 1, p. 35-43, 2001.

MIODUSKI, J.; MORO, R. S. Grupos funcionais da vegetação campestre de Alagados,Ponta Grossa, Paraná. Iheringia, Botânica, Porto Alegre, v. 66, n. 2, p. 241-256, 2011.

MOBOT. MISSOURI BOTANICAL GARDEN. (W33Tropicos) Nomenclatural data base. 2013. Disponível em: <http://www. mobot.org/w3 search/vast.html>. Acesso em: 3 nov. 2013.

MONTESINOS-TUBÉE, D. B. Diversidad florística de la cuenca alta del río Tambo-Ichuña (Moquegua, Perú). Revista Peruana de Biología, Lima, v. 18, n. 1, p. 119-132, 2011.

MUELLER-DOMBOIS, D.; ELLENBERG, H. Aims and methods of vegetation ecology. New York: John Willey \& Sons, 1974. 547 p. NICÁCIO, J. E. M. A Manutenção de mata ciliar: um ativo permanente. Revista de Estudos Sociais, Campo Grande, v. 3, n. 6, p. 85-92, 2001.

PARAÍBA. Agência Executiva de Gestão das Águas do estado da Paraíba. Monitoramento dos volumes dos Açudes. 2013. Disponível em: <http://site2.aesa.pb.gov.br/aesa/volumesAcudes. do>. Acesso em: 12 dez. 2013.

PEDRALLI, G.; TEIXEIRA, M. C. B. Reservatórios: alternativas para proteção das comunidades vegetais na sua área de influência. Ensaios e Dissertações, São Paulo, v. 5, n. 1, p. 34-41, 1997.

PEREIRA, O. J.; ASSIS, A. M. Florística da restinga de Camburi, Vitória, ES. Acta Botanica Brasilica, Porto Alegre, v. 14, n. 1, p. 99-111, 2000.

PIŠEK, P.; RICHARDSON, D. M.; REJMÁNEK, M.; WEBSTER, G. L.; WILLIAMSON, M.; KIRSCHNER, J. Alien plants in checklists and floras: towards better communication between taxonomists and ecologists. Taxon, Vienna, v. 53, n. 1, p. 131-143, 2004.

PIVARI, M. O. D.; VIANA, P. L.; LEITE, F. S. F. The Aquatic macrophyte flora of the Pandeiros River wildlife sanctuary, Minas Gerais, Brazil. Check List, Rio Claro, v. 9, n. 2, p. 415-424, 2013.

RAUNKIAER, C. The life forms of plants and statistical plant geography. Oxford: Claredon, 1934. $632 \mathrm{p}$.

RIBEIRO-FILHO, A. A.; FUNCH, L. S.; RODAL, M. J. N. Composição florística da floresta ciliar do rio Mandassaia, Parque Nacional da Chapada Diamantina, Bahia, Brasil. Rodriguésia, Rio de Janeiro, v. 60, n. 2, p. 265-279, 2009.

SCUDELLER, V. V.; SOUZA, A. M. G. Florística da mata de igapó na Amazônia Central. In: SANTOS-SILVA, E. N.; SCUDELLER, V. V. (Ed.). Biotupé: meio físico, diversidade biológica e sociocultural do baixo Rio Negro, Amazônia Central. Vol. 2. Manaus: UEA Edições, 2009. 302 p.
SHAN-HUAH, W. U. TSAI, J. K.; SUN, H. T.; CHEN, C. F.; CHIOU, C. R. Patterns of plant invasions in the preserves and recreation areas of Shei-Pa National Park in Taiwan. Botanical Studies, Taipei, v. 50, n. 1, p. 217-227, 2009.

SILVA, R. K. S.; FELICIANO, A. L. P.; MARAGON, L. C.; LIMA, R. B. A.; SANTOS, W. B. Estrutura e síndromes de dispersão de espécies arbóreas em um trecho de mata ciliar, Sirinhaém, Pernambuco, Brasil. Revista Pesquisa Florestal Brasileira, Colombo, v. 32, n. 69, p. 1-11, 2012.

SOUZA, A.; ARAÚJO, R. R.; ARROIO-JR, P. P.; LEAL, A. C. Situação atual da mata ciliar de um trecho do rio Santo Anastácio, SP. Tópos, Presidente Prudente, v. 5, n. 2, p. 182-197, 2011.

SOUZA, C. G.; SANTOS, F. S.; CUNHA, I. S.; MENEZES, M. V.; ARAÚJO, T. S. A Degradação da Cobertura Vegetal do município de Vitória da Conquista - BA. Enciclopédia Biosfera, Goiânia, v. 6, n. 1, p. 1-15, 2008.

SPRENT, J. I. Legume trees and shrubs in the tropics: Nz fixation in perspective. Soil Biology \& Biochemistry, Oxford, v. 27, n. 4, p. 401-407, 1995.

SUHOGUSOFE, V. G.; PILIACKAS, J. M. Breve histórico da ação antrópica sobre os ecossistemas costeiros do Brasil, com ênfase nos manguezais do estado de São Paulo. Integração, São Paulo, v. 51, n. 1, p. 343-351, 2007.

SWEENEY, B.; BOTT, T.; JACKSON, J.; KAPLAN, L.; NEWBOLD, J.; STANDLEY, L.; HESSION, W.; HORWITZ, R. Riparian deforestation, stream narrowing and loss of stream ecosystem services. Proceedings of the National Academy of Sciences of the United States of America, Washington, v. 101, n. 39, p. 14132-14137, 2004.

THE PLANT LIST. A working list of all plants species. 2013. Disponível em: <http://www.theplantlist.org/>. Acesso em: $12 \mathrm{dez}$. 2013.

UNITED NATIONS. Kyoto protocol to the United Nations framework convention on climate change. 1998. Disponível em: $<$ http://www.unfecc.int/>. Acesso em: 8 fev. 2013.

VELOSO, H. P.; OLIVEIRA-FILHO, L. C.; VAZ, A. M. S. F.; LIMA, M. P. M.; MARQUETE, R.; BRAZÃO, J. E. M. Manual técnico da vegetação brasileira. Rio de Janeiro: Fundação do Instituto Brasileiro de Geografia e Estatística (IBGE), 1992. 92 p.

VOGEL, H. F.; ZAWADZKI, C. H.; METRI, R. Florestas ripárias: importância e principais ameaças. SaBios: Revista de Saúde e Biologia, Campo Mourão, v. 4, n. 1, p. 24-30, 2009.

ZEPEDA-GÓMEZ, C.; LOT-HELGUEIRAS, A.; NEMIGA, X. A.; MADRIGAL-URIBE, D. Florística y diversidad de las ciénegas del rio Lerma estado de México, México. Acta Botánica Mexicana, Patzcuaro, v. 98, n. 1, p. 23-49, 2012.

ZIPPARRO, V. B.; GUILHERME, F. A. G.; ALMEIDA-SCABBIA, R. J.; MORELLATO, L. P. C. Levantamento florístico de floresta atlântica no sul do estado de São Paulo, Parque Estadual Intervales, Saibadela. Biota Neotropica, Campinas, v. 5, n. 1, p. 147-170, 2005. 